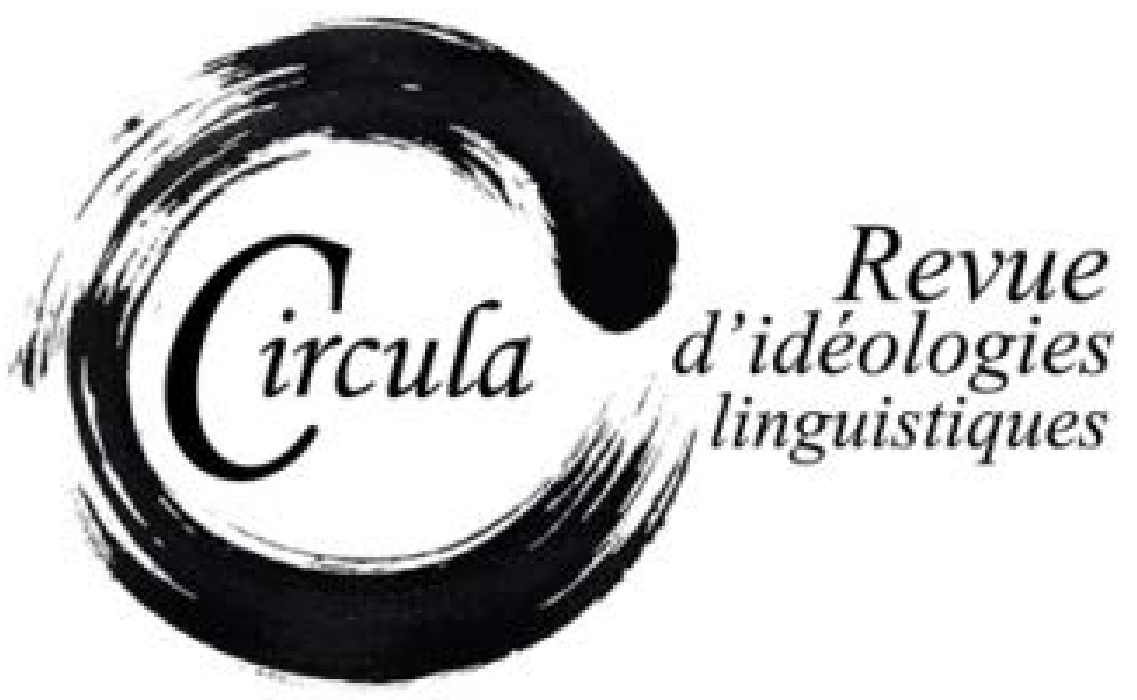

TITRE: LA PERSISTANCE DE L'IDÉOLOGIE LINGUISTIQUE DES REMARQUEURS DANS LES CHRONIQUES DE LANGAGE DE 1925 À NOS JOURS

Auteur(s): Wendy Ayres-BenNett, University of CAMBridge

Revue: Circula, NumÉRo 1, PAGES 44-68

ISSN: 2369-6761

DiRecteurs: Wim REMYSEn et SABINE SCHWARZE

URI: HTTP://HDL.HANDLE.NET/11143/7992

DOI: $10.17118 / 11143 / 7992$ 


\title{
La persistance de l'idéologie linguistique des remarqueurs dans les chroniques de langage de 1925 à nos jours
}

\author{
Wendy Ayres-Bennett, University of Cambridge \\ wmb1001@cam.ac.uk
}

Résumé : Dans cet article j'examine dans quelle mesure l'idéologie linguistique des remarqueurs du XVII siècle, et notamment celle exprimée par Claude Favre de Vaugelas dans ses célèbres Remarques sur la langue françoise de 1647, persiste dans les chroniques de langage publiées en France ou en Belgique depuis les années 1920. Ces courts articles sur l'usage du français qui apparaissent dans les journaux ou revues ont joué un rôle important dans la presse française au XX des chroniques de trois moments représentatifs dans l'histoire de ce genre : les années 1920 et 1930 ; la période 1950-1970, considérée par certains comme «l'âge d'or » du genre ; et les quinze dernières années. Je m’intéresse en particulier à la persistance de certaines notions clés - la pureté, la netteté ou la clarté, et la conception de ce qui constitue le bon usage du français.

Mots-clés : remarques sur la langue française; chroniques de langage ; pureté ; netteté ; bon usage

Abstract: In this article I examine the extent to which the linguistic ideology of the remarqueurs, and notably that expressed by Claude Favre de Vaugelas in his famous Remarks on the French Language of 1647, persists in newspaper and magazine columns on the French language published in France or Belguim since the 1920s. These short articles on the use of French have played an important role in the French press in the twentieth century. I analyse the French language columns from three representative periods in the history of the genre: the 1920s and 1930s; 1950-1970, considered by some as the "Golden Age" of the genre; and the last fifteen years. I consider in particular the persistence of certain key concepts - purity, precision or clarity, and the concept of good French usage.

Keywords: remarks on the French language; newspaper and magazine columns on language usage; purity; clarity; good usage 


\section{Introduction}

Dans cet article je voudrais examiner dans quelle mesure les chroniques de langage publiées dans les journaux français et belges depuis les années 1920 sont toujours imprégnées de l'idéologie linguistique des remarqueurs. Je commencerai par présenter brièvement les deux genres avant de considérer l'intérêt de les comparer.

\section{Deux genres métalinguistiques: les chroniques de langage et les remarques sur la langue}

\subsection{Qui sont les remarqueurs?}

Une série de volumes d'observations et de remarques sur la langue française parut dans la deuxième moitié du XVII siècle, suite à la publication des Remarques sur la langue françoise de Claude Favre de Vaugelas en $1647^{1}$. Ces recueils de courtes remarques détachées traitent de subtilités ou d'idiotismes de l'usage qui posaient problèmes à ceux qui parlaient le mieux la langue française à l'époque. Parmi les remarqueurs les plus connus, citons Dominique Bouhours (1692 [11675], 1693 [11692]), Gilles Ménage (1675 [ $\left.\left.{ }^{1} 1672\right], 1676\right)$, et Nicolas Andry de Boisregard (1692 [11689], 1693) ${ }^{2}$. Pour cette étude je me suis surtout appuyée sur les quinze volumes qui constituent le Corpus des remarques sur la langue française (XVII' siècle) (Ayres-Bennett, 2011).

\subsection{Chroniques de langage}

Quant aux chroniques de langage ou chroniques de grammaire qui ont paru dans la presse écrite, c'est un genre qui a connu un grand succès au XXe siècle dans le monde francophone. Je me concentrerai ici sur la presse française et belge, mais comme l'a bien montré Wim Remysen (2011, 2012, 2013), entre autres, le genre jouit également d'une production abondante et d'une large diffusion au Canada.

La publication de chroniques ne se limite pas aux journaux nationaux. Elles ont paru également dans les quotidiens provinciaux de grande diffusion et dans les magazines et revues telles que les Nouvelles Littéraires, le Figaro Littéraire, le Rail, le Carrefour, le Télé-Septjours et les Lectures pour tous,

1. M. Doug Kibbee a récemment « découvert» le texte de Jean de Wepy (ou Wapy), publié à Mousson en 1634, qui porte le titre Les Remarques sur la langue françoise pour parler à la mode et selon l'air du temps. À de nombreux égards, cet ouvrage ressemble aux remarques de Vaugelas et est peut-être à considérer comme l'un des textes fondateurs du genre. Nous avons fait une première présentation de ce texte au $13^{\text {th }}$ International Conference on the History of the Language Sciences à Vila Real, Portugal, 25-29 août 2014.

2. Nous citons la dernière édition du vivant de l'auteur. Les détails bibliographiques des textes du corpus sont donnés dans Ayres-Bennett (2011). 
«sans oublier le combatif et vigilant bulletin de Défense de la langue française aux destinées duquel préside Paul Camus » (Georgin, 1965 : 4).

Parmi les chroniqueurs, on trouve des hommes de lettres - par ex. André Thérive (Les Nouvelles Littéraires, Carrefour, Le Temps, etc.), Abel Hermant (Le Temps) - et des linguistes ou professionnels de la langue - Albert Dauzat (Le Monde), Robert Le Bidois (Le Monde), Marcel Cohen (Les Étoiles, L'Humanité), Maurice Grevisse (Le Soir). Selon Jacques Cellard (1983: 652), qui succéda à Robert Le Bidois au Monde 3 , il ne s'agit jamais d'un journaliste professionnel.

Plusieurs critiques ont parlé du déclin du genre en France. Par exemple, Jacques Cellard a écrit en 1983:

Le genre est à éclipses. Les années 1930-1935 en ont marqué une apogée, les années 19601965 une autre ; les présentes en sont un périgée, au moins en France, car la presse belge se montre plus constante et la presse québécoise plus intéressée. (Cellard, 1983 : 653)

Pour expliquer ce déclin, il fait mention de raisons commerciales, du fossé entre la linguistique et « la philologie simplette de nos pères », et de l'immense diffusion du Bon Usage de Grevisse en France.

Selon Charles Muller, ce sont plutôt les années 1950 qui constituent « l'âge d'or » des chroniques, âge d'or qui s'achève en 1970. Il note :

Au milieu de notre siècle, l'esprit de Vaugelas s'est brillamment incarné dans une génération de chroniqueurs du langage ; leur nombre, modeste en 1950, avait quadruplé en peu d'années. (Muller, 2004 : 243)

Pour lui, la décadence du genre s'explique par le fait que « dans l'après-mai-68, toute autorité, même langagière, est suspecte, et l'arbitrage des remarqueurs n'est plus de saison » (Muller, 2004 : 244-245).

René Georgin, écrivant en 1965, semble également se concentrer sur deux périodes : la période entre deux guerres, où ont paru les chroniques de Maurice Schöne, de Victor Snell, d'André Thérive et d'André Moufflet, entre autres (Georgin, 1965 : 4) ; et l'époque contemporaine, où les chroniques «se sont multipliées. [...] [L]e mouvement se propage du Monde à France-Soir, du Figaro à l'Aurore, de la Croix à l'Humanité ».

3. Pour une collection de ses chroniques, voir Cellard (1979). 
Si on a plusieurs fois sonné le glas pour le genre, il continue à y avoir des exemples intéressants à la fin du XXe siècle et même au début de notre siècle :

- «Le bon français » dans Le Figaro - Bochnakowa (2005) examine 1267 billets signés de 32 auteurs pour la période 1996-2000 ; parmi ces chroniqueurs figure Maurice Druon de l'Académie française (Druon, 1999) qui a instigué la rubrique ; il aurait voulu la participation des Académiciens, mais seul Jean Dutourd y a contribué

- Alain Bladuche-Delage, La Croix (2003)

- Claude Duneton, Figaro littéraire (2004)

- Alain Rey, «La vie des mots » dans le Magazine littéraire (Rey et Maja, 2009, etc.)

De nos jours le genre n'est pas limité à la presse écrite : il y a des chroniques de langage à la radio (par ex. Alain Rey, France Inter) et à la télévision (par ex. Bernard Cerquiglini, TV5). Le genre continue également à travers l'internet et les blogs : par exemple, Alain Bladuche-Delage écrit un blog intitulé "L'encre bleu $»^{4}$ et les correcteurs du Monde rédigent celui intitulé «Langue sauce piquante $»^{5}$. Notons aussi les rapports avec les guides d'usage. En 2012, Bernard Cerquiglini a publié un volume de chroniques qui n'ont pas paru dans la presse, mais qui traitent des même questions épineuses : la différenciation de mots apparentés, le genre des substantifs, la féminisation de noms de métier, les pièges de l'orthographe, les exigences de la prononciation, etc.

Dans les chroniques, on trouve l'idée que les médias sont en partie responsables du mauvais état du français, et c'est donc par les médias qu'il convient de l'améliorer :

Que la langue française, au long des dernières décennies, n’ait cessé de se dégrader est une évidence. Il est une évidence aussi que la plupart des fautes de langage, impropriétés, barbarismes, solécismes, négligences des accords, prononciations boiteuses, inventions de néologismes inutiles ou obscurs, emplois abusifs de vocables étrangers ou dérivations absurdes de ces vocables sont commis ou propagés par les médias. [...] C'est par et à travers les médias qu'il convient d'endiguer le mal, s'il se peut. (Druon, $1999: 7$ )

Comme nous verrons ci-dessous, cette citation contient plusieurs termes clés des deux traditions. Pour Druon (1999 : 235), ce sont les médias oraux - et notamment la télévision - qui sont avant tout coupables de la diffusion du mauvais français : «Car la télévision participe de plus en plus à la destruction de notre langage, tandis que la presse écrite contribue, vaille que vaille, à son maintien ».

4. http://www.encre-bleue.com/spip.php?article139.

5. http://correcteurs.blog.lemonde.fr/. 
L'un des intérêts du genre est qu'il nous donne des informations sur ce que Françoise Gadet (1999 : 643) appelle « la pesanteur des mentalités » qui n'évoluent que peu au fil du temps 6 . De plus, nous avons une sorte de « dialogue », car les chroniqueurs répondent souvent à l'abondant courrier des lecteurs. En étudiant les quelques 3000 lettres envoyées à Félicien Mars (pseudonyme de Gaëtan Monfet), qui a publié des chroniques entre 1952 et 1982 dans La Croix, quotidien catholique à diffusion nationale, Claude Haezebrouck (1988) nous donne un aperçu précieux des préoccupations linguistiques des lecteurs de ce journal'?

Pour illustrer mon argument, j'ai pris mes exemples chez les chroniqueurs représentatifs de trois moments de l'évolution du genre:

1. Pour la période la plus ancienne : André Thérive (pseudonyme de Roger Puthoste) (1929) et Abel Hermant (Lancelot) (1936, 1938), académicien, « quintessence du genre normatif sans la moindre sensibilité au mouvement de la langue » (Gadet, 1999 : 644)

2. Pour la période dite d'« âge d'or » : Marcel Cohen (1950) ; Maurice Grevisse (1961-1964)', ainsi que quelques exemples de chroniqueurs catalogués dans la bibliographie de Quemada $(1970,1972)$ pour la période 1950-1970

3. Pour les quinze dernières années : Druon (1999), Bladuche-Delage (2003), Duneton (2004) et Cerquiglini (2008)

Il faut toutefois noter que ni les remarqueurs ni les chroniqueurs ne constituent un groupe homogène.

\subsection{Pourquoi faire cette comparaison?}

Comme l'ont déjà remarqué plusieurs études (cf. Schwarze, 1977; Ayres-Bennett, 1987; Caron, 2004), il y a des parallélismes évidents entre les deux genres qui s'opèrent à plusieurs niveaux. Anna Bochnakowa (2013), tout en hésitant à appeler les chroniqueurs du Figaro (ou d'autres journaux) des «remarqueurs», me semble toutefois offrir une description des chroniqueurs qui convient également aux remarqueurs:

[...] nous préférons les considérer comme des observateurs, vigilants, sensibles ou sensibilisés aux faits de langue et faisant partager leurs considérations et, pourquoi pas, leurs émotions parfois, à l'égard du français. (Bochnakowa, 2013: 172-173)

6. Voir aussi Osthus (2005), qui situe les chroniques de langage dans la tradition de la «Laienlinguistik» (linguistique populaire ou profane).

7. Il note, par exemple, que près de $35 \%$ des lettres concernent la langue de la presse.

8. Ce sont les trois premiers volumes d'une série de cinq. 
Les similarités ont été reconnues parles chroniqueurs eux-mêmes. Bernard Cerquiglini (2008:5) dans l'introduction de ses chroniques note qu'on peut identifier deux traditions dans l'histoire des idées linguistiques en France : ce qu'il appelle la grammaire française et les remarqueurs. II continue:

Si la seconde tradition est moins illustre, elle est amplement illustrée. On n’en finirait pas d'établir la liste des publications qui, avec un talent divers, se placèrent dans le sillage de Vaugelas. [...] Jusqu'à ces dernières années, pas de grand quotidien national qui n'eût sa «chronique de langage ». (Cerquiglini, $2008: 7$ )

[...] On voit quel genre littéraire s'est ainsi constitué au cours des siècles ; les émissions Merci professeur! n'ayant pas d'autre ambition que d'en être une illustration contemporaine. Il convient de faire bref (quelques paragraphes), d'user d'une langue accessible (les termes techniques indispensables sont expliqués) et d'une expression plaisante; une des lois du genre semble être la «chute» finale qui joue avec le terme étudié. (Cerquiglini, 2008: 9)

Avant de considérer si les deux genres partagent une même idéologie linguistique, je pointerai quelques similarités de forme et de contenu.

\subsubsection{Forme et présentation}

Du point de vue de la forme, les observations des remarqueurs sont le plus souvent assez courtes, la longueur variant de quelques lignes à quelques pages. Elles s'enchaînent pêle-mêle sans organisation ou ordre logique. Les raisons pour lesquelles Vaugelas a adopté cette présentation pour ses Remarques de 1647 sont élaborées dans la douzième section de sa Préface. Il rejette l'ordre alphabétique qui ne facilite pas la compréhension des remarques et il refuse également l'ordre fondé sur les neuf parties du discours - qu'il aurait choisi s'il avait voulu écrire une grammaire formelle - parce que cet ordre ne servirait qu'à ceux qui savent la langue latine et par conséquent les termes grammaticaux. En revanche, ce qu'il appelle « cét agreable meslange » non seulement lui permet d'ajouter de nouvelles observations jusqu'au moment de la publication du texte, mais possède aussi de bonnes qualités intrinsèques, «car il est certain que cette continuelle diversité de matieres recrée l'esprit, et le rend plus capable de ce qu'on luy propose, sur tout quand la briefveté y est jointe, comme icy, et qu'on est asseuré que chaque Remarque fait son effet ». L'absence de tout fil conducteur entre de courtes remarques détachées se retrouve dans les recueils de Bouhours (1692, 1693), de Ménage (1675, 1676), et de Tallemant (1698). Quelques-uns préfèrent adopter un ordre alphabétique (Alemand, 1688; Andry, 1692, 1693) ou regrouper les remarques dans de grands chapitres thématiques (Buffet, 1668), mais les observations restent ponctuelles.

9. Un texte instructif par rapport à ces questions est la chronique programmatique de René Georgin (1965), intitulée "Qu'est-ce qu'une chronique de grammaire?». 
Par définition, les chroniques de langage sont de courtes discussions d'un problème linguistique particulier. Même quand elles sont recueillies plus tard dans un livre, on garde habituellement la présentation décousue. Par exemple, dans la préface à son premier volume de Querelles de langage (1929), André Thérive observe:

On trouvera d'ailleurs dans ce livre non pas un traité, mais un répertoire où j’ai voulu laisser l'aspect du désordre et de la variété. [...] Des fautes ou des erreurs que présentent des livres renommés pour leur nouveauté ou leur valeur, des cas litigieux de vocabulaire ou de syntaxe, des références à des exemples ou des ouvrages mal connus, tels sont les titres de ce livre à la curiosité et peut-être à l'agrément. (Thérive, 1929 : préface non paginée)

De la même façon, Marcel Cohen (1950) note dans ses Regards sur la langue française:

Aucun ordre n'était suivi de manière voulue; l'actualité et des questions de lecteurs ont influé sur le choix des matières; quelques regroupements ont été faits dans le présent recueil, mais sans introduire de plan systématique. (Cohen, 1950 : 11)

Et Claude Duneton (2004 : 11) continue dans la même tradition, même si son ouvrage est organisé en quatre grands chapitres thématiques; son recueil est "comme un florilège de ma production hebdomadaire. [...] Il faut jouer le jeu : j’ai laissé les textes dans l'état où ils ont paru dans le journal, sans modification ».

Deuxième chose à remarquer en ce qui concerne la présentation : dans les deux genres, le contenu est adapté à un public de non-spécialistes. Le lectorat désigné implicitement par les remarqueurs se constitue des honnêtes gens français qui fuient le pédantisme et qui ne connaissent pas nécessairement la grammaire latine. Les chroniques, comme nous l'avons déjà vu, paraissent souvent dans des journaux de grande circulation et leur contenu est adapté au grand public.

Par conséquent, les auteurs évitent l'usage de termes trop techniques. Comme il s'adresse à un public d'honnêtes gens, Vaugelas juge préférable, au moins en théorie, d'éviter les termes techniques et la terminologie de la grammaire formelle. Cela reflète également une tendance générale de l'époque qui consiste à ne pas employer de termes techniques ou spécialisés dans le discours poli :

Les termes de l'art sont tousjours fort bons et fort bien receus dans l'estenduë de leur jurisdiction, où les autres ne vaudroient rien; et le plus habile Notaire de Paris se rendroit ridicule, et perdroit toute sa pratique, s'il se mettoit dans l'esprit de changer son stile, et ses phrases pour prendre celles de nos meilleurs Escrivains; Mais aussi que diroit-on d'eux s'ils escrivoient, Iceluy, jaçoit que, ores que, pour et à icelle fin, et cent autres semblables que les Notaires employent? (Vaugelas, 1647 : Préface IX, 3) 
Georgin (1965 : 4) décrit la façon dont Abel Hermant (Lancelot) « [enrobait] d’une sauce agréable une sauce piquante - les discussions techniques arides ». Duneton (2004: 10) observe que le public des chroniques est particulièrement varié ; pour cette raison, «il faut rédiger sans trop d'ostentation, en évitant les pesanteurs - ce qui, sur ces sujets langagiers, n'est pas facile. II convient même de montrer une pointe d'humour - un journal n'est pas un traité de linguistique ». Bref, Cellard (1983: 653) observe qu'il s'agit d'« un savoir pratique et plaisant».

\subsubsection{Contenu}

Du point de vue du contenu, le point de départ pour les remarqueurs est un doute sur une question de bon usage ou l'examen de l'« usage douteux ». Vaugelas annonce cette approche dès la première page de sa préface :

Mon dessein n'est pas de reformer nostre langue, ny d'abolir des mots, ny d'en faire, mais seulement de monstrer le bon usage de ceux qui sont faits, et s'il est douteux ou inconnu, de l'esclaircir, et de le faire connoistre. Et tant s'en faut que j'entreprenne de me constituer Juge des differens de la langue, que je ne pretens passer que pour un simple tesmoin, qui depose ce qu'il a veu et oüi, ou pour un homme qui auroit fait un Recueil d’Arrests qu'il donneroit au public. (Vaugelas, 1647 : Préface I)

Les chroniqueurs traitent également des « hésitations de l'usage ». On lit, par exemple, sur la quatrième de couverture de l'ouvrage d'Alain Bladuche-Delage (2003) :

Bladuche-Delage, du journal la Croix, a sélectionné pour nous un florilège de ses brillantes chroniques hebdomadaires qui mettent l'accent sur ce qu'il convient d'appeler les hésitations de l'usage - vous savez, ces mots a priori très simples dont on connaît tous l'emploi... mais qui se révèlent truffés d'embûches!

De la même façon, Bernard Cerquiglini (2008) parle de problèmes concrets que la langue pose à son usager, et observe:

Moins qu'une théorie de la langue, le francophone hésitant attend une réponse, explication limpide venant infirmer ou conforter son opinion. Car, et ceci explique enfin le succès des remarqueurs, tout francophone porte en lui un sentiment de la langue. (Cerquiglini, 2008: 8)

Les ressemblances vont pourtant plus loin, car les deux groupes se concentrent sur les mêmes types de questions. Les remarqueurs s'intéressent surtout aux questions de lexique et de morphosyntaxe. Dans le tableau 1, je présente la proportion de remarques consacrées à chaque thématique dans sept volumes de remarques (de Vaugelas [1647], Ménage [1675, 1676], Bouhours [1692, 1693] et Andry de Boisregard $[1692,1693])^{10}$. Cette analyse montre que le pourcentage moyen de remarques

10. Pour plus d'informations, voir Ayres-Bennett et Seijido (2011), chapitre 3. 
consacrées au lexique est de ca. 55 \% et à la morphosyntaxe de ca. $30 \%$. En revanche, il y a relativement peu de remarques sur la prononciation et l'orthographe : seulement ca. 7 \% des remarques dans nos sept volumes concernent la prononciation et ca. $2 \%$ l'orthographe.

Tableau 1 : Préoccupations des remarqueurs et des chroniqueurs

\begin{tabular}{|c|c|c|}
\hline Domaine & Remarqueurs (7 volumes) & Chroniqueurs (ex. de Druon, 1999) \\
\hline Lexique & ca. $55 \%$ & ca. $55 \%$ \\
\hline Morphosyntaxe & ca. $30 \%$ & ca. $20 \%$ \\
\hline Prononciation & ca. $7 \%$ & ca. $3 \%$ \\
\hline Orthographe & ca. $2 \%$ & ca. $5 \%$ \\
\hline
\end{tabular}

Chez les chroniqueurs la prononciation et l'orthographe sont également des intérêts mineurs (par ex. chez Druon [1999] il s'agit de $3 \%$ et $5 \%$ respectivement). Viennent ensuite les problèmes de morphosyntaxe (20\% chez Druon). Les observations consacrées au lexique sont nettement majoritaires, comme chez les remarqueurs (ca. $55 \%$ chez Druon).

On remarque aussi une étonnante continuité dans les préoccupations des deux groupes. Dans le domaine de la morphosyntaxe, par exemple, on constate quelques centres d'intérêt importants tels que les questions du genre et des accords du verbe, l'usage de prépositions et les constructions verbales. Comme le note Cerquiglini (2008):

Le sujet des chroniques est en partie imposé : un dépouillement du recueil publié par Bernard Quemada, les questions posées par les téléspectateurs sur le site de TV5 font apparaître une thématique assez stable : l'accord du participe passé, le genre de amour, délice et orgue, la signification exacte d'achalandé, la différence entre second et deuxième, l'emploi de septante et nonante, etc. : nous n'avons pas manqué d’y répondre. (Cerquiglini, 2008 : 9-10)

\section{Idéologie linguistique}

Passons maintenant aux questions d'idéologie linguistique ${ }^{11}$. Dans ce court article je ne peux traiter que de trois concepts clés qui se retrouvent chez les remarqueurs et les chroniqueurs : le bon usage, la pureté, et la netteté ou la clarté.

11. Jacques Cellard (1983: 664) décrit le chroniqueur comme « directeur de consciences linguistiques », description qui me semble également convenir aux remarqueurs. 


\subsection{Bon usage}

Dans un premier temps, j'examinerai la définition et le rôle du bon usage chez les remarqueurs et les chroniqueurs. Je considérerai ensuite la question de savoir si les remarqueurs et les chroniqueurs sont prescriptifs.

La notion de «bon usage » se trouve au cœur de la doctrine de Vaugelas qu'il définit de la façon suivante: "C'est la façon de parler de la plus saine partie de la Cour, conformément à la façon d'escrire de la plus saine partie des Autheurs du temps » (Vaugelas, 1647 : Préface II, 3). Lorsque ni la Cour, ni les auteurs ne peuvent résoudre une question, Vaugelas fait appel à une troisième autorité, les « gens sçavants en la langue », référence implicite à l'Académie française.

Plusieurs éléments de la définition sont à remarquer :

- L'emploi de l'expression « la plus saine partie » indique qu'il s'agit de l'usage d'une élite ; Vaugelas note en outre que: "Selon nous, le peuple n'est le maistre que du mauvais Usage, et le bon Usage est le maistre de nostre langue ». (Vaugelas, 1647 : Préface VII, 3)

- La définition du bon usage reflète le contexte socioculturel dans lequel Vaugelas vivait : il favorise l'usage de la Cour royale, dont l'essor préfigurait déjà l'émergence de la monarchie absolue de Louis XIV.

- Vaugelas s'intéresse autant à l'usage parlé qu'à l'usage écrit. Ses successeurs, pourtant, comme Bouhours et Andry de Boisregard, se concentrent de plus en plus sur l'usage écrit.

La promotion du bon usage fait partie de la mission du chroniqueur et est représentée dans les trois périodes que j'ai étudiées. II n'est peut-être pas surprenant que l'on trouve l'une des discussions les plus élaborées du bon usage dans le premier volume des Problèmes de langage de Maurice Grevisse, auteur de la grammaire de référence, Le Bon Usage. Grevisse se situe explicitement dans le sillon de Vaugelas. Dans la première chronique du recueil intitulée «Considérations sur l'usage », il nous offre la réponse suivante à la question « Sur quoi faut-il [...] se fonder pour décider si telle façon de dire est « correcte » ou si elle ne l'est pas?»:

On l'oublie parfois : en matière de correction du langage, la grande règle, la règle infaillible est celle de l'usage. Qu'on y prenne garde : cela ne signifie nullement que telle expression est bonne parce que tout le monde l'emploie. Il y a, en effet, un usage vulgaire, des manières de parler ou d'écrire (provincialismes, termes argotiques ou populaires, expressions boutiquières, langage d’Allobroge...) qui, tout en offrant à la linguistique ou à la grammaire simplement descriptive des faits souvent fort intéressants, n'ont à retenir l'attention des amateurs du bon langage que pour être l'objet de leurs condamnations. 
À cet usage des «nombreux» (comme disait Abel Hermant), c'est-à-dire au mauvais usage, il convient d'opposer le bon usage - et nous voilà ramenés à l'excellent Vaugelas, pour qui ce bon usage était « la façon de parler de la plus saine partie de la Cour, conformément à la façon d'escrire de la plus saine partie des Autheurs du temps ». En adaptant à l'époque moderne cette formule célèbre, il est aisé de définir le bon usage actuel, qui est le consentement des bons écrivains et des gens qui ont souci de bien s'exprimer. (Grevisse, 1961 : 5-6)

L'article fait plusieurs échos à Vaugelas; par exemple l'usage, qui ne suit pas toujours la logique, est considéré comme « le maître souverain de la langue » (Grevisse, 1961 : 10), et, en ce qui concerne le changement linguistique, communis error facitjus (Grevisse, 1961 : 8). Comme chez les remarqueurs, l'usage de bons auteurs sert de patron ; mais Grevisse se différencie en incluant dans sa liste d'autorités non seulement des auteurs « du temps » mais aussi ceux du XVII et du XVIII ${ }^{e}$ siècle $e^{12}$.

La notion du bon usage est aussi très présente dans les chroniques plus récentes. Comme le note Anna Bochnakowa (2013 : 173), le titre même de la rubrique du Figaro, « Le bon français », représente la continuation de l'idée d'une langue d'élite. Cette notion est par exemple évidente dans les billets de Maurice Druon, qui insiste sur la distinction entre le bon et le mauvais usage. Pour lui, l'Académie française est « le greffier» du bon usage (Druon, 1999: 251) ${ }^{13}$.

Haezebrouck (1988) observe le même intérêt pour la norme dans les lettres envoyées à Félicien Mars entre 1952 et 1982. Selon ses recherches, plus de 60 \% des lettres font référence à la norme (c'est-àdire incluent une interrogation sur la norme, la défense de la norme, le pointage des « fautes », etc.).

La grande différence par rapport à la position de Vaugelas est l'absence d'autorité attribuée à la langue parlée. Au contraire, plusieurs chroniqueurs partagent le préjugé selon lequel la langue parlée est considérée comme très fautive. Paul Camus (1966 : 5) soutient que le rôle de la presse, de la radio et de la télévision dans la diffusion rapide de ce qu'il appelle « les fautes de syntaxe et les impropriétés de vocabulaire », " ne nous permet plus de laisser évoluer la langue, mais [...] rend au contraire indispensable une définition unique du bon usage et une discipline propre à le faire respecter».

Si les remarqueurs et les chroniqueurs prônent le bon usage, sont-ils donc prescriptifs ? Notre recherche en collaboration avec Magali Seijido (Ayres-Bennett et Seijido, 2011) montre que les remarqueurs font preuve de deux idéologies qui coexistent dans leurs ouvrages. D'une part, on peut identifier une tendance prescriptive qui vise à promouvoir le bon usage et à rejeter les variantes considérées comme représentatives du mauvais usage. Les remarques qui s'inscrivent dans ce modèle contiennent souvent une expression déontique telle qu'«il faut dire » :

12. Seul Gilles Ménage, dans ses deux volumes d'observations $(1675,1676)$, cite fréquemment l'usage des anciens auteurs.

13. Cette position fait également écho à Vaugelas (1647 : Préface I) qui se présente comme simple témoin de l'usage et non pas comme juge. 
Il faut dire, midy est sonné, dix heures sont sonnées, et non, a sonné : l'horloge a sonné, et non, est sonné, parce que c'est l'horloge qui sonne les heures, et que ce sont les heures qui sont sonnées par l'horloge. (Andry de Boisregard, 1692: 640-641)

Dans la remarque intitulée "Arrivé qu'il fut, arrivé qu'il estoit, marri qu'il estoit », la première phrase indique nettement l'attitude prescriptive de Vaugelas :

Toutes ces façons de parler ne valent rien, quoy qu'une infinité de gens s'en servent, et en parlant, et en escrivant. Au lieu de dire, arrivé qu'il fut, arrivé qu'il estoit, il faut dire, estant arrivé, il exprime tous les deux, ou bien, comme il fut arrivé, comme il estoit arrivé. Et au lieu de marri qu'il estoit, il faut dire, estant marri, ou marri, tout seul. Ce qui apparemment est cause d'une phrase si mauvaise, c'est que nous en avons d'autres en nostre langue, fort approchantes de celle-là, qui sont tres-bonnes et tres-elegantes [...]. (Vaugelas, 1647 : 139)

D'autre part, dans d'autres remarques, on peut identifier une autre idéologie selon laquelle l'usage est considéré comme roi, et qui accepte l'usage de deux ou plusieurs constructions ou mots. Dans ce modèle de nature « sociolinguistique », le remarqueur nous offre une analyse plus nuancée selon laquelle les usages sont plus ou moins bons selon le contexte, le registre ou le style. Par exemple, à propos du choix entre «sur les armes, et sous les armes » Vaugelas conseille qu' :

il faut user tantost de l'un et tantost de l'autre, afin qu'il ne semble pas que l'on condamne celuy dont on ne se sert jamais, en quoy l'on auroit tort, et pour conserver d'ailleurs tout ce qui contribuë à la richesse de nostre langue; comme est de pouvoir dire une mesme chose de deux façons, plustost que d'une seule. (Vaugelas, 1647 : 396-397)

Dans la Préface de ses Réflexions (1692), Andry de Boisregard adopte également une position ouverte à la variation sociolinguistique :

Les termes y [c.-à-d. dans ces réflexions] sont marquez chacun selon leur caractere propre, et plusieurs y sont appellez bas et populaires, sans qu'on pretende pour cela les condamner : Car tous les mots ont leur place : souvent il est à propos de se servir d'expressions communes, selon la nature du sujet; quelquefois mesmes elles donnent de la force aux choses.

D’ailleurs nôtre Langue abonde en toutes sortes de façons de parler, elle en a pour le stile mediocre, et pour le sublime; pour le serieux, et pour le burlesque; Il faut tâcher d'en faire le discernement : et c'est en quoy consiste presque toute la science des paroles. (Andry de Boisregard, 1692 : préface non paginée)

Il faut en outre noter qu'un langage prescriptif peut cacher une bonne description de l'usage actuel qui est en train de se modifier. Prenons le cas de la remarque de Vaugelas intitulée «Print, prindrent, prinrent ». Notre remarqueur observe: 
Tous trois ne valent rien, ils ont esté bons autrefois, et M. de Malherbe en use tousjours, Et d'elle prindrent le flambeau, dont ils desolerent leur terre, etc. Mais aujourd'huy l'on dit seulement, prit, et prirent, qui sont bien plus doux. (Vaugelas, 1647 : 98)

Si l'on regarde l'usage de la première moitié du siècle, comme il est attesté dans la base Frantext ${ }^{14}$, on observe que la remarque de Vaugelas se vérifie.

Qu'en est-il des chroniqueurs? Ici encore on trouve beaucoup de variation. Abel Hermant adopte en général une position prescriptive. Dans sa définition du rôle de chroniqueur, René Georgin met aussi l'accent sur l'obligation du chroniqueur de proscrire les mauvais usages et de prescrire les bons. Sa fonction principale est donc « de lutter contre les malfaçons diverses qui menacent le français, soit en prévenant le lecteur, soit en répondant à ses questions» (Georgin, 1965 : 6).

Quant aux chroniqueurs des quinze dernières années, ils se montrent également plus ou moins prescripteurs. En faisant l'analyse des chroniques du Figaro, Anna Bochnakowa (2013 : 173-174) constate qu'on en trouve différents types. Il continue à y avoir celles qui adoptent un ton prescriptif ou qui font un jugement de valeur non seulement sur un fait de langue observé mais aussi sur les locuteurs représentant un usage précis. Typiques de ce genre sont les chroniques de Maurice Druon; par exemple, dans sa chronique sur débuter et démarrer, il maintient:

L’Académie a maintes fois rappelé que le verbe débuter n'admet pas de complément d'objet direct. Il est donc tout à fait fautif de dire d'un orateur qu'il débute son discours : il le commence. (Druon, 1999 : 11)

Autre exemple : en commentant l'usage de l'expression « voix off » et l'alternative proposée par l'Académie « voix hors champ », il exprime le regret que, « [c]omme il arrive souvent, la prescription [soit] venue un peu tard, et la mauvaise habitude persiste encore » (Druon, 1999 : 79). Anna Bochnakowa remarque toutefois qu'il existe d'autres chroniques qui montrent un caractère didactique et neutre. Tandis que sa description de la rubrique semble établir une distinction entre les chroniqueurs et vaugelas, il me semble que sa caractérisation des chroniqueurs du Figaro convient également aux remarqueurs:

Si l'on reprend le sens que Vaugelas avait donné au «bon usage », il faudrait voir dans le titre de la rubrique la continuation de l'idée d'une langue d'élite, groupe social bien déterminé et, pour l'époque contemporaine, celui des gens cultivés, des intellectuels conscients de la qualité d'une certaine norme ou plutôt d'un modèle idéal du français. Mais en même temps, les mêmes chroniqueurs remarquent bien les tendances de l'évolution de la langue, ainsi que de ses variétés quand ils présentent, voire condamnent ou ridiculisent certains usages. De ce point de vue, les billets du Figaro donnent aussi un tableau du français actuel, varié et en mouvement. (Bochnakowa, 2013 : 173)

14. http://www.frantext.fr/. Pour plus d'informations, voir Ayres-Bennett (à paraître). 
De la même façon, si Bernard Cerquiglini semble commencer par associer une attitude prescriptive à la fois au remarqueur et au chroniqueur, la dernière phrase de la citation fait preuve d'une attitude plus souple:

Contrainte plus délicate, une attitude s'impose au remarqueur, celle de la prescription. On attend de lui qu'il distingue, qu'il précise, qu'il réprouve ; le purisme n'est jamais loin. Libéral en matière de langue par profession, le linguiste devenu chroniqueur se confronte à la norme. Sans rien abjurer de ce qu'il sait ni de ce qu'il pense, il lui faut convenir qu'une norme (même souple) s'impose, que la langue gagne à être précise, que les mots ont un sens. II se fera donc prescripteur, mais avec le sourire (d'où la « scie » initiale : « Ne craignons pas d'être un peu puriste ; à bon escient du moins ») et non sans intention. [...] Changé en remarqueur, le linguiste a mis un Grevisse dans sa poche, mais pas son drapeau : il plaide pour les rectifications orthographiques proposées par le Conseil supérieur de la langue française avec l'accord de l'Académie française ; il milite en faveur de la féminisation des noms de métier, titres et fonctions. Il rappelle enfin que la langue française, langue mondiale, tire sa force tout autant de sa diversité et de sa plasticité que de sa rigueur. (Cerquiglini, 2008: 10-11)

\subsection{Pureté}

Je traiterai plus rapidement des qualités principales qui sont invoquées pour caractériser le bon usage du français. À la fin de son volume Vaugelas offre une série de remarques qui définissent et exemplifient deux catégories centrales de sa pensée linguistique, la pureté et la netteté :

La pureté du langage et du stile consiste aux mots, auxphrases, auxparticules, et en la syntaxe.

Et la netteté ne regarde que l'arrangement, la structure, ou la situation des mots, et tout ce qui contribuë à la clarté de l'expression. (Vaugelas, $1647: 567$ )

La pureté et la netteté sont ici expliquées avant tout par l'énumération des façons de les contrevenir. Après avoir examiné l'importance de la pureté de l'expression chez les remarqueurs et les chroniqueurs, je considérerai s'il convient de les caractériser comme puristes.

\subsubsection{Pureté : barbarismes et solécismes}

Les fautes contre la pureté sont les barbarismes et les solécismes que Vaugelas (1647 : 567-577) définit de sa propre façon. Les barbarismes « des mots » et « des phrases » concernent pour la plupart le choix correct d'un mot ou d'une expression; il s'agit d'éviter tout terme qui n'est « pas François », qui ne s'emploie pas dans un sens donné, qui n'est plus en usage ou qui est trop nouveau. II inclut toutefois certaines questions syntaxiques dans sa liste de barbarismes, par exemple l'usage d'un adverbe pour une préposition. Dans l'énumération des types de solécismes, Vaugelas fait mention de différents types de problèmes linguistiques : les erreurs de déclinaison et de conjugaison ainsi que « toutes les fautes qui se commettent contre les reigles de la syntaxe ». La liste comprend des 
questions relatives au choix du genre des noms, à la forme correcte des pronoms, à l'accord des participes, à l'accord avec les noms dits collectifs, à la construction des prépositions, etc.

Notons que dès les années 1930, Abel Hermant utilise le même métalangage pour critiquer certains usages du français. Jacques Cellard offre les exemples suivants pris des chroniques du Temps:

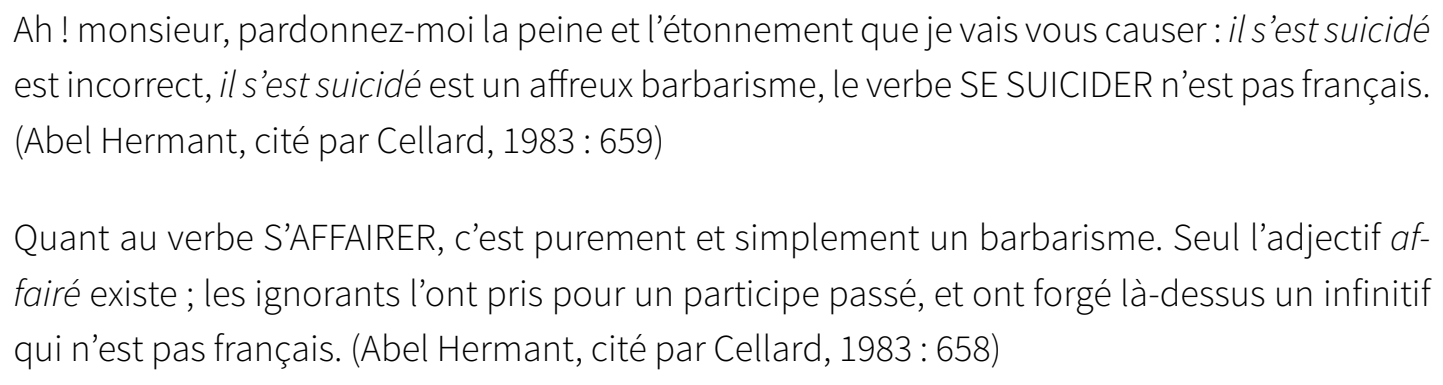

Quant au verbe S'AFFAIRER, c'est purement et simplement un barbarisme. Seul l'adjectif affairé existe ; les ignorants l'ont pris pour un participe passé, et ont forgé là-dessus un infinitif qui n'est pas français. (Abel Hermant, cité par Cellard, 1983: 658)

Maurice Druon cite l'opinion de Rivarol selon laquelle «La langue française n'eut donc que deux sortes de barbarie à combattre : celle des mots et celle du mauvais goût de chaque siècle » (Druon, 1999 : 193) ; selon lui, le XXe siècle a battu tous les records de faiblesse " contre l'invasion des mots barbares et la prolifération du mauvais goût ». Et les néologismes du type de réenvironnementaliser ou de recontextualiser entendus sur France-Inter « sont des attentats à la langue française » (Druon, 1999 : 219).

\subsubsection{Pureté et propriété des mots et des phrases (expressions)}

Si nous regardons la liste des barbarismes élaborée par Vaugelas, il semble que la pureté est liée à une autre notion clé des remarqueurs, celle de la propriété des mots et des phrases, qui, selon Laurent Chiflet, dans son Essay d'une parfaite grammaire de la langue françoise de 1659, consiste «à se servir des mots et des phrases propres au sujet que l'on traite » (Chiflet, 1659 : 148).

La propriété peut concerner soit l'acceptabilité des termes de façon absolue (les termes sont rejetés ou acceptés sans nuance), soit la relativisation ou la restriction de l'usage selon la variation sociolinguistique. Tous les remarqueurs s'intéressent beaucoup au choix du mot juste et sont par conséquent très préoccupés par l'acceptabilité des termes, par leur bon usage sémantique, et par la différenciation de termes apparentés. Quant à la variation sociolinguistique, ils s'attachent surtout à la discussion de mots nouveaux ou vieillis, mais ils traitent également de la variation selon le registre, le genre et parfois selon la région.

Les chroniqueurs sont également très sensibles aux nuances de l'emploi du mot juste. Les remarques et chroniques qui appartiennent à cette rubrique sont très nombreuses, et je me limite ici à quelques exemples représentatifs. 


\section{a) Sens précis des mots et des expressions}

Beaucoup d'observations des remarqueurs précisent le sens des mots et les contextes où l'on peut les employer :

Onguent pour parfum.

Un fameux Autheur est repris, et avec raison, d'avoir escrit onguent, en parlant de la Magdeleine, et dit un precieux onguent, au lieu d'un precieux parfum. Nous avons encore plusieurs de nos Escrivains et de nos Predicateurs, qui font cette faute. Ce qui les trompe, c'est que les Latins disent unguentum, en cette signification, parce que les Anciens se servoient de certains parfums, comme il y en a encore de plusieurs sortes parmy nous, dont le vray usage estoit de s'en oindre quelques parties du corps ; tellement qu'il semble qu'on avoit raison de l'appeller onguent. Mais parce que ce mot se prend tousjours pour medicament, il ne s'en faut jamais servir pour parfum, l'Usage le veut ainsi. (Vaugelas, 1647 : * 458)

AlLiage.

Cét alliage de Lettres, dit l'Auteur de l'Art de Parler, augmente les alphabets d'un grand nombre de consonnes. Alliage n'est pas bon là, il ne se dit gueres que des métaux. (Andry de Boisregard, 1692: 47)

Le chroniqueur, Maurice Druon, censure les nouveaux usages comme l'extension sémantique du verbe risquer:

La saison, lit-on parfois, risque d'être belle.

Voilà un risque que l'on devrait plutôt considérer comme une chance.

Si l'on risque d'échouer, on a pourtant des chances de succès.

En revanche, la langue française risque de pâtir de ces emplois absurdes, où l'on fait perdre aux mots leur vrai sens. (Druon, 1999 : 19)

On enjoint aux lecteurs d'éviter un terme défavorisé tel que dimension et de choisir plutôt un mot plus juste:

Si l'on souhaite écrire un honnête français, on se demandera, chaque fois que l'on est tenté d'employer dimension, si les mots aspect, portée ou caractère ne seraient pas plus justes, et donc plus élégants. (Druon, 1999: 76) 


\section{b) Différenciation de termes apparentés}

Dans cette recherche du mot juste, les remarqueurs différencient souvent des termes apparentés de forme et/ou de sens:

Temperature, Temperament.

Ces deux mots ont deux usages bien differens, il ne les faut pas confondre. Temperature se dit de l'air, et temperament des personnes. Il faut que le Medecin sçache le temperament du malade, c'est à dire la complexion du malade; Car je ne parle pas de temperament en un autre sens pour adoucissement. Toutefois M. de Malherbe use de temperature pour temperament. M. le Cardinal de Lorraine, dit-il, fut d'une temperature, où il n'y avoit rien à desirer. Je l'ay veu aussi employé tout de mesme dans Amyot. Mais c'est, qu'il se disoit autrefois, et il ne se dit plus. (Vaugelas, $1647: 74)$

\section{VACATIONS, VACANCES.}

Vacations se dit pour le Palais, vacances pour le Collége. Les Avocats étudient durant les vacations; les Ecoliers perdent le tems durant les vacances. M. Pelisson dit pourtant: Pendant que nous étions au Collége mon frére et moi, on nous permettoit d'aler passer tout le tems des vacations à la campagne. Mais il y a bien de l'aparence que M. Pelisson avoit oublié le Collége, et les termes du Collége, quand il se mit à écrire l'Histoire de l'Academie Françoise. (Bouhours, $1692: 131)$

Selon Maurice Rat, « le juste emploi des mots dans la seule propriété de leur sens contribue à la clarté de la langue. [...] Suranné n'est pas l'équivalent de désuet, ni constatation de constat, ni constance de patience » (Rat, 1965 : 604). Il énumère plusieurs « faux frères » : acceptation et acception, agonir et agoniser, conjecture et conjoncture, imposer et en imposer, rabattre et rebattre, recouvrer et recouvrir, somptuaire et somptueux, suggestion et sujétion, de suite et tout de suite. Les mêmes termes sont discutés par différents chroniqueurs; Cerquiglini (2008) écrivant plus de quarante ans après Rat, reprend, par exemple, la discussion du sens d'acception:

Acception.

Ne craignons pas d'être un peu puriste; à bon escient du moins.

Je n'aime pas que l'on confonde l'acceptation et l'acception. [...] (Cerquiglini, $2008: 21$ ) 


\subsubsection{Pureté vs purisme}

Il est important ici de faire une distinction nette entre la promotion d'un langage pur et une attitude puriste ${ }^{15}$. Selon Olivia Walsh (2012: 4), le purisme est caractérisé par les éléments suivants :

- Seule une forme de la langue est correcte ou même « parfaite » (en général la langue standard)

- Cette langue est pure et, par conséquent, toute modification représente une contamination ou une corruption

- $\quad$ Il est essentiel de protéger la langue contre toute contamination et de la préserver dans son état actuel (ou, si la langue commence déjà à être corrompue, il faut éliminer les éléments corrompus)

II me semble que les remarqueurs et les chroniqueurs ne constituent pas un groupe homogène de ce point de vue. Tous les remarqueurs s'intéressent à décrire l'usage actuel du français qu'ils distinguent souvent de celui des « anciens écrivains ». À mon avis, Vaugelas n'adopte pas une position puriste, car il admet que la langue se modifiera dans une période de 25 ou 30 ans, même si les principes qui guident le bon usage perdureront. Environ un cinquième de ses remarques traitent de la variation diachronique et de l'établissement de l'usage actuel. Au début du siècle suivant, les Académiciens font des observations sur les remarques de Vaugelas dont le but est souvent de les mettre à jour (Académie française, 1704).

L'attitude des remarqueurs envers les néologismes est également nuancée. Il se trouve qu'ils censurent les néologismes:

Invectiver.

Invectiver, pour faire des invectives, n'est pas du bel usage, et il n'est pas permis de faire des verbes à sa fantaisie, tirez et formez des substantifs. Beaucoup de gens neantmoins se donnent cette authorité ; mais il n'y a que les verbes, que l'Usage a receus, dont on se puisse servir, sans qu'il y ayt en cela ny reigle, ny raison. Par exemple on dit, affectionner, se passionner, d'affection et de passion, et plusieurs autres semblables, et neantmoins si l'on veut bien parler, on ne dira pas ambitionner, occasionner, d'ambition, et d'occasion, non plus que pretexter, pour prendre pretexte, et se medeciner pour prendre medecine. Je sçay bien qu'ils sont en la bouche de la pluspart du monde; mais non pas dans les escrits des bons Autheurs. (Vaugelas, 1647 : 119-120)

15. Le sens de ces deux termes s'est évidemment modifié au cours des siècles. Sur l'histoire de ces notions dans le domaine roman, voir Ludwig et Schwarze (2006), qui montrent comment le concept de purisme en France a progressivement acquis des connotations négatives. II me semble que l'usage du verbe « affecter » et de l'expression adverbiale « trop scrupuleusement » dans la définition du « puriste » dans la première édition du dictionnaire de l'Académie française de 1694 ( On appelle ainsi un homme qui affecte la pureté du langage, et qui s’y attache trop scrupuleusement ») implique déjà que le purisme se caractérise par l'excès. 
Il faut noter toutefois que les mots nouveaux peuvent aussi être acceptés s'ils sont établis :

Intrigue.

La pluspart font ce mot feminin, je dis la pluspart, parce qu'il y en a qui le font de l'autre genre; il faut dire intrigue, avec un $g$, et non pas intrique, avec un $q$, comme force gens le disent et l'escrivent. C'est un nouveau mot pris de l'Italien, qui neantmoins est fort bon, et fort en usage. (Vaugelas, $1647: 126)$

INACTION.

Inaction est un terme nouveau, mais qui est fort bon et fort en usage. «Le travail d'une promenade, parce qu'il est utile à la santé, plaist davantage que l'inaction. » (Andry de Boisregard, $1692: 259-260)$

Quant aux chroniqueurs, ils se montrent plus ou moins puristes ${ }^{16}$. Abel Hermant fait preuve d'un purisme extrême :

ATTIRANCE est « une horrible préciosité ». (Abel Hermant, cité par Cellard, 1983 : 658).

Pourquoi pas, tant que vous y êtes, le difforme verbe INTENSIFIER? Intensif est un terme soit de scolastique, soit d'agriculture; dans la conversation où il s'est abusivement glissé, c'est un terme à la fois impropre et puant de pédanterie. (Abel Hermant, cité par Cellard, 1983:658)

Une chronique de Maurice Rat de décembre 1965 censure également certains néologismes:

On peut aussi enfouir au «musée des horreurs » des néologismes aussi monstrueux qu'alluré (qui a de l'allure), arbragé (planté d'arbres), attenté (qui a été victime d'un attentat), conversationniste (brillant dans la conversation), minoré (dont on a diminué l'importance), moratorié (bénéficiaire d'un moratoire), relationné (qui a de hautes relations), urgencer (hâter). (Rat, 1965 : 603)

Le linguiste Marcel Cohen (1950), d'autre part, est beaucoup plus ouvert au changement linguistique. Discutant du néologisme solutionner pour résoudre, il commente:

Et la vérité, ici, c’est que les prétendus monstres sont en réalité des produits naturels et légitimes, qu'ils ne sont pas des fantaisies individuelles plus ou moins coupables, mais qu'ils se rangent dans des séries et des systèmes [...]. (Cohen, 1950 : 9)

16. Selon Gadet (1999: 639), au XXe siècle, « [l]es offensives puristes connaissent des moments plus vigoureux : le tournant du siècle (bataille de l'orthographe, sur fond de confrontation entre tenants de deux types de société) ; les années 30 (crise économique internationale, danger de guerre) ; les années 60 (constat de persistance de l'échec scolaire, affaiblissement du rôle international de la France) ». 
Cellard (1983: 662) observe que les chroniqueurs sont préoccupés par les changements d'usage ${ }^{17}$. Il conclut: " Il existe donc bien un "champ" de la chronique du langage : c'est celui des tensions entre la norme et l'usage montant» (Cellard, 1983: 663).

Georgin (1965: 6) note qu'on a tendance à abuser du mot puriste. Il exprime toutefois lui-même une position puriste quand il prétend qu'

On ne saurait nier, en effet, que la langue française d'aujourd'hui, sauf chez quelques grands prosateurs, a perdu, par comparaison avec celle du XIXe siècle et même du début du XXe', de sa pureté et de sa propriété et que les règles de la grammaire traditionnelle y sont souvent violées. (Georgin, 1965:5).

Druon parle même du rôle que peut jouer le chroniqueur en arrêtant l'établissement des néologismes:

Je suis parfaitement reconnaissant à ceux de mes lecteurs qui veulent bien me signaler, dès qu'ils apparaissent, les néologismes fâcheux, inutiles, choquants ou absurdes. Pour leur barrer le chemin, il faut non seulement être attentif à les relever, mais rapide à les dénoncer. Car sinon, en six mois ils s'installent, et après deux ans on nous fera valoir qu'ils sont dans l'usage. (Druon, 1999: 127)

\subsection{Netteté}

Vaugelas (1647 : 577-585) énumère et exemplifie également les fautes contre la netteté : la « mauvaise situation des mots ", la "mauvaise structure » (dans ces cas « il y a tousjours quelque chose à ajouster, ou à diminuer, ou à changer non pas simplement pour le lieu, mais pour les mots »), les syntagmes coordonnés mal construits, la séparation de deux membres d'une période par une autre période qui fonctionne comme une parenthèse entre eux, et les périodes trop longues. II faut avant tout éviter les constructions équivoques.

Selon André Thérive (1929 : préface) : « Le principal péril que nous courions aujourd'hui, c’est de ne pas écrire assez clair, assez simple ». La clarté du français - ou plutôt le mythe de la clarté du français ${ }^{18}$ - est examinée par plusieurs chroniqueurs, parmi lesquels figurent Albert Dauzat (1952) et Maurice Rat (1965). Après avoir considéré les raisons pour lesquelles le français est plus clair que d'autres langues, Dauzat conclut:

17. Christoph Schwarze (1977: 31) considère que le changement du titre de la rubrique dans Le Monde à partir de 1972 de "La défense de la langue française » à « La vie de langage » est symbolique de l'adoption d'une attitude moins normative. 18. Voir, par exemple, le chapitre de Lodge intitulé « French is a Logical Language» dans Bauer et Trudgill (1998 : 23-31). 
La clarté du français impose des devoirs à ceux qui le parlent, plus encore à ceux qui l'écrivent: respecter la propriété des termes et surtout respecter la syntaxe, cette architecture de la langue, dont tous les éléments sont solidaires, et qu'on ne saurait disloquer sans porter atteinte à l'édifice. (Dauzat, 1952: 7)

Maurice Rat prétend que ce qu'il appelle « une syntaxe de plus en plus lâche, qui tend à substituer dans la langue écrite les approximations ou les pataquès de la langue parlée » (Rat, 1965 : 605), constitue l'une des plus grandes menaces à la clarté du français. Dans une chronique intitulée « Des maladies actuelles du langage » publiée en novembre 1967 dans la Défense de la langue française, il ajoute que « [l]a syntaxe [...] est ébranlée de telle sorte que l'équivoque n'a jamais autant nui à une langue qui fut longtemps claire» (Rat, 1967 : 4).

Dans la pratique, les exemples de constructions équivoques ou ceux qui concernent l'ordre des mots sont relativement rares dans les chroniques. Maurice Grevisse (1964) dans sa chronique intitulée «Séparer la préposition de son régime ? », qui cite l'opinion de Vaugelas, note qu'il existe quelques exemples littéraires de cette séparation, mais qu'il n'en reste pas moins que :

l'usage normal et ordinaire est de ne pas séparer la préposition de son régime et qu'à vouloir imiter des exemples tels que ceux qui viennent d'être cités, nous manquerions généralement de bonnes occasions d'être naturels et d'écrire avec simplicité [...]. (Grevisse, 1964 : 354-355)

En général, pourtant, les chroniqueurs préfèrent traiter des questions syntaxiques telles que l'accord du verbe avec des mots collectifs ou le choix de mode après certains verbes et prépositions ${ }^{19}$.

\section{Conclusion}

Il est évident qu'il ne faut pas exagérer les parallélismes entre les remarqueurs et les chroniqueurs. $\mathrm{Ni}$ les remarqueurs ni les chroniqueurs ne forment un groupe entièrement homogène et ils peuvent se montrer plus ou moins puristes, plus ou moins prescriptifs, etc. De plus, il existe des différences importantes entre les remarqueurs et les chroniqueurs, puisque leurs ouvrages linguistiques reflètent le contexte socioculturel dans lequel ils furent publiés ${ }^{20}$. Nous avons toutefois observé que plusieurs éléments clés de l'idéologie des remarqueurs subsistent dans la presse écrite française des XXe et

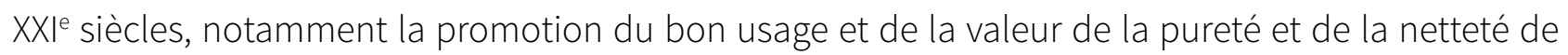
langage.

19. Le fait qu'il y ait relativement peu de chroniques dédiées aux problèmes concernant l'ordre des mots ou l'anaphore suggère peut-être que les références fréquentes à la clarté du français font partie d’une stratégie rhétorique ou discursive.

20. Il existe peut-être également des différences entre leur influence respective. Si les remarqueurs ont exercé une influence à la fois sur les auteurs contemporains et sur la tradition grammaticale ultérieure (voir Ayres-Bennett et Seijido, 2011 : 253-268), il est plus difficile d'identifier des résultats tangibles de l'influence des chroniqueurs. Comme nous l'avons remarqué, il est frappant d'observer dans quelle mesure les mêmes questions sont traitées par les générations successives de chroniqueurs. 


\section{Références}

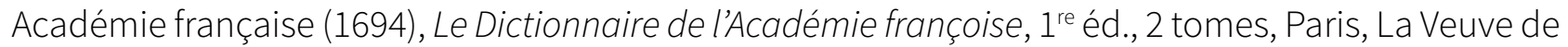
J. B. Coignard et J. B. Coignard.

Académie française (1704), Observations de l'Académie françoise sur les Remarques de M. de Vaugelas, Paris, J. B. Coignard.

Alemand, Louis-Augustin (1688), Nouvelles Observations, ou Guerre civile des François, sur la langue, Paris, J.-B. Langlois.

Andry de Boisregard, Nicolas (1692), Réflexions, ou Remarques critiques sur l'usage present de la

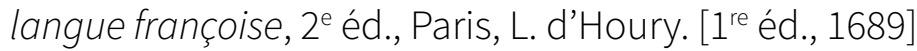

Andry de Boisregard, Nicolas (1693), Suite des Réflexions critiques sur l'usage present de la langue françoise, Paris, L. d'Houry.

Ayres-Bennett, Wendy (1987), Vaugelas and the Development of the French Language, Londres, Modern Humanities Research Association.

Ayres-Bennett, Wendy (dir.) (2011), Corpus des remarques sur la langue française (XVIIe siècle), Paris, Classiques Garnier Numérique.

Ayres-Bennett, Wendy (à paraître), "Codification and Prescription in Linguistic Standardisation : Myths and Models », dans Francesc Feliu (dir.), Constructing Languages : Norms, Myths and Emotions, Amsterdam, John Benjamins.

Ayres-Bennett, Wendy et Magali Seijido (2011), Remarques et observations sur la langue française: histoire et évolution d'un genre, Paris, Classiques Garnier.

Bauer, Laurie et Peter Trudgill (dir.) (1998), Language Myths, Londres, Penguin.

Bladuche-Delage, Alain (2003), Ici ? ou là ? Les traîtres mots, Paris, Éditions Mots \& Cie et la Croix.

Bochnakowa, Anna (2005), Le bon français de la fin du XXe siècle : chroniques du «Figaro » 1996-2000, Cracovie, Wydawnictwo Uniwersytetu Jagiellońskiego.

Bochnakowa, Anna (2013), "Chroniques de langage dans Le Figaro (1996-2000) », dans Wendy Ayres-Bennett et Magali Seijido (dir.), Bon usage et variation sociolinguistique : perspectives diachroniques et traditions nationales, Lyon, ENS Éditions, p. 171-177.

Bouhours, Dominique (1692), Remarques nouvelles sur la langue françoise, $3^{e}$ éd., Paris, G. et L. Josse. [1 éd., 1675]

Bouhours, Dominique (1693), Suite des Remarques nouvelles sur la langue françoise, Paris, G. et L. Josse. [1 ${ }^{\text {re }}$ éd., 1692] 
Buffet, Marguerite (1668), Nouvelles Observations sur la langue françoise, où il est traitté des termes anciens et inusitez, et du bel usage des mots nouveaux. Avec les éloges des illustres sçavantes, tant anciennes que modernes, Paris, J. Cusson.

Camus, Paul (1966), « Un état fort peut imposer une discipline du langage », Défense de la langue française, n 32 (avril 1966), p. 3-6.

Caron, Philippe (dir.) (2004), Les remarqueurs sur la langue française du XVIe siècle à nos jours, Rennes, Presses Universitaires de Rennes.

Cellard, Jacques (1979), La vie du langage : chroniques 1971-1975 «Le Monde», Paris, Le Robert.

Cellard, Jacques (1983), « Les chroniques de langage », dans Édith Bédard et Jacques Maurais (dir.), La norme linguistique, Québec/Paris, Conseil de la langue française/Le Robert, p. 651-666.

Cerquiglini, Bernard (2008), Merci professeur! Chroniques savoureuses sur la langue française, Paris, Bayard.

Cerquiglini, Bernard (2012), Petites chroniques du français comme on l'aime!, Paris, Larousse.

Chiflet, Laurent (1659), Essay d'une parfaite Grammaire de la langue françoise Ou le Lecteur trouvrera, en bel ordre, tout ce qui est de plus necessaire, de plus curieux, et de plus elegant, en la Pureté, en l'Orthographe, et en la Prononciation de cette Langue, Anvers, J. van Meurs.

Cohen, Marcel (1950), Regards sur la langue française, Paris, Sedes.

Dauzat, Albert (1952), « Pourquoi le français est-il une langue claire? », Le Monde, 6 août 1952, p. 7.

Druon, Maurice (1999), Le «Bon Français », Monaco, Éditions du Rocher.

Duneton, Claude (2004), Au plaisir des mots, Paris, Éditions Balland.

Gadet, Françoise (1999), «La langue française au XXe siècle. 1. L'émergence de l'oral », dans Jacques Chaurand (dir.), Nouvelle histoire de la langue française, Paris, Seuil, p. 583-671.

Georgin, René (1965), « Qu'est-ce qu'une chronique de grammaire? », Défense de la langue française, n³ 30 (décembre 1965), p. 4-9.

Grevisse, Maurice (1961-1964), Problèmes de langage, 3 vol., Paris, Presses universitaires de France.

Haezebrouck, Claude (1988), «L'insécurité linguistique des Français à travers le courrier des lecteurs de La Croix (1952-1982), Recherches sur le français parlé, n 9, p. 39-52.

Hermant, Abel (1936), Chroniques de Lancelot du «Temps », Paris, Larousse.

Hermant, Abel (1938), Chroniques de Lancelot du «Temps», tome II, Paris, Larousse.

Ludwig, Ralph et Sabine Schwarze (2006), « Die Vorstellung sprachlicher "Reinheit" in der Romania: von der stilistischen Pragmatik zur Symbolik einer nationalen und supranationalen Kultur », dans Sabine Schwarze et Edeltraud Werner (dir.), Identitätsbewahrung und Identitätsbegründung durch Sprache. Aktuelle Beiträge zum frankophonen Raum, Hamburg, Verlag Dr. Kovač, p. 3-33. 
Ménage, Gilles (1675), Observations de Monsieur Ménage sur la langue françoise, 2e éd., Paris, C. Barbin. [1'éd., 1672]

Ménage, Gilles (1676), Observations de Monsieur Ménage sur la langue françoise. Segonde partie, Paris, C. Barbin.

Muller, Charles (2004), «Les années cinquante : l'âge d'or des remarqueurs », dans Philippe Caron (dir.), Les remarqueurs sur la langue française du XVIe siècle à nos jours, Rennes, Presses Universitaires de Rennes, p. 243-250.

Osthus, Dietmar (2005), « Laienlinguistik und Sprachchroniken : Französisch und Okzitanisch », dans Gerhard Ernst, Martin-Dietrich Gleßgen, Christian Schmitt et Wolfgang Schweickard (dir.), Romanische Sprachgeschichte/Histoire linguistique de la Romania : manuel international d'histoire linguistique de la Romania, vol. 2, Berlin/New York, Walter de Gruyter, p. 1533-1546.

Quemada, Bernard (1970), Bibliographie des chroniques de langage publiées dans la presse française 1 : 1950-1965, Paris, Didier.

Quemada, Bernard (1972), Bibliographie des chroniques de langage publiées dans la presse française 2 : 1966-1970, Paris, Didier.

Rat, Maurice (1965), «Clarté française », La revue des deux mondes, 15 décembre 1965, p. 602-605.

Rat, Maurice (1967), « Des maladies actuelles du langage », Défense de la langue française, n 40 (novembre 1967), p. 3-4.

Remysen, Wim (2011), «L'application du modèle de l'Imaginaire linguistique à des corpus écrits : le cas des chroniques de langage dans la presse québécoise », Langage et société, n 135, p. 47-65.

Remysen, Wim (2012), « Les représentations identitaires dans le discours normatif des chroniqueurs de langage canadiens-français depuis le milieu du XIX siècle », Journal of French Language Studies, n²2, p. 419-445.

Remysen, Wim (2013), «Comment définir le bon usage au Canada français ? Le point de vue des chroniqueurs de langage », dans Wendy Ayres-Bennett et Magali Seijido (dir.), Bon usage et variation sociolinguistique : perspectives diachroniques et traditions nationales, Lyon, ENS Éditions, p. 179-193.

Rey, Alain et Daniel Maja (2009), À bas le génie! Et autres chroniques décalées, Paris, Fayard.

Schwarze, Christoph (1977), Sprachschwierigkeiten, Sprachpflege, Sprachbewusstsein : das Phänomen der "Chroniques de langage», Konstanz, Universität Konstanz.

Tallemant, Paul (1698), Remarques et decisions de l'Académie françoise, Paris, J.-B. Coignard.

Thérive, André (1929), Querelles de langage, [vol. 1], Paris, Librairie Stock, Delamain et Boutelleau.

Vaugelas, Claude Favre de (1647), Remarques sur la langue françoise utiles à ceux qui veulent bien parler et bien escrire, Paris, Veuve J. Camusat et P. le Petit. 
Walsh, Olivia (2012), Linguistic Purism in France and Quebec, thèse de doctorat, Cambridge, Université de Cambridge.

Wepy (ou Wapy), Jean de (1634), Les Remarques sur la langue françoise pour parler à la mode et selon l'air du temps, Mousson, Philippe Vincent. 